\title{
Cardiovascular Diseases and the Common Risk Factors Presented by Patients at Kitwe Teaching Hospital
}

\author{
Mwanza Josphat ${ }^{1}$, Sichilima Matafwali Alfred ${ }^{2}$ \\ ${ }^{1}$ Department of Public Health, Micheal Chilufya Sata School of Medicine, Copperbelt University, Ndola, Zambia \\ ${ }^{2}$ Department of Biological Sciences, School of Mathematics and Natural Sciences, Copperbelt University, Kitwe, Zambia
}

Email address:

mwanzajosphat@yahoo.com (M. Josphat), alflima.mata@gmail.com (S. M. Alfred)

\section{To cite this article:}

Mwanza Josphat, Sichilima Matafwali Alfred. Cardiovascular Diseases and the Common Risk Factors Presented by Patients at Kitwe Teaching Hospital. Rehabilitation Science. Vol. 4, No. 1, 2019, pp. 1-6. doi: 10.11648/j.rs.20190401.11

Received: October 23, 2019; Accepted: May 7, 2019; Published: June 5, 2019

\begin{abstract}
Cardiovascular disease (CVD) refers to any disease that affects the cardiovascular system, such as cardiac disease, vascular diseases of the brain and kidney and peripheral arterial diseases. CVDs are among the leading causes of morbidity and mortality in Zambia. A case-control study was conducted among 74 patients suffering from cardiovascular disease (CVD) at Kitwe Teaching Hospital (KTH) and 74 controls (patients without CVD). All participants completed a questionnaire regarding socio-demographics, risk factors of CVD and Knowledge based questions of CVD. Chi-square test was used to determine significant associations and a result yielding a $\mathrm{p}$ value of less than $5 \%$ was considered statistically significant. Independent factors associated with development of CVD were established using a Binary Logistic Regression. Unadjusted odds ratio (OR) and Adjusted odds ratios (AOR) and their 95\% confidence intervals (CI) are reported. The independent risk factors which were significantly associated with the development of CVD were hypertension (OR 59.143, 95\% CI 20.122-173.833; AOR 97.289, 95\% CI 24.479 -386.662), diabetes mellitus (OR 3.725, 95\% CI $1.722-8.057$; AOR 6.033, 95\% CI 1.630 -22.332), family history (OR 2.504, 95\% CI 1.242-5.050; AOR 0.862, 95\% CI 0.244 -3.041), smoking (OR 1.587, 95\% CI 0.731-3.448; AOR 0.746, 95\% CI 0.164 -3.403), alcohol consumption (OR 1.387, 95\% CI 0.725 -2.654; AOR 3.392, 95\% CI 0.833 - 13.823), high blood cholesterol (OR 4.364, 95\% CI 0.894 - 21.293; AOR 4.482, 95\% CI 0.242- 82.888) and increased age (P value: 0.039). Three factor were significantly associated with reduced risk of developing CVD. These were physical activity (OR 0.575, 95\% CI 0.287-1.150; AOR 1.849, 95\% CI 0.507 - 6.751), balanced diet (OR 0.890, 95\% CI 0.456-2.195; AOR 1.451, $95 \%$ CI $0.404-5.214$ ) and regular weight check and control (OR 0.897, 95\% CI $0.471-1.710$; AOR $0.593,95 \%$ CI 0.177 1.990). Interventions such as proper control of hypertension and diabetes pharmacologically or non - pharmacologically, as well as providing health education on life style risk factors that are associated with CVD may significantly reduce morbidity and mortality due to the disease.
\end{abstract}

Keywords: Kitwe Teaching Hospital, Cardiovascular Disease, Non-Communicable Disease

\section{Introduction}

Non-Communicable Diseases, particularly cardiovascular diseases have become a major public health problem across the world. Developing countries, including Zambia, are currently undergoing an epidemiological transition, from "Communicable or Infectious" to "Non-Communicable" diseases, these are rapidly growing with major consequences on morbidity and mortality [1]. At Kitwe teaching hospital morbidity and mortality due to CVD and hypertension is on the increase. In 2016, 494 cases of CVD were recorded of which 261 patients were admitted. In the same year, 120 deaths due to CVD alone were recorded. In 2017, the number of cases recorded increased to 583 and so did the number of deaths due to the disease (168 deaths were recorded). The 2012 Annual Health Statistical Bulletin by Zambia's Ministry of Health, revealed that there was a $22 \%$ increase in the total number of NCDs cases between 2010 and 2012 in all age groups (MOH, 2014) as cited [2]. The burden of cardiovascular disease is estimated to be twice as high in 
2020 as it was in 1999 with severe negative impact on the economic development [3]. In Kuwait, it was found that the most common risk factors of CVD known by more than three-quarters of respondents were smoking, obesity and unhealthy diet. Age, family history of CVD, regular eating of healthy food and BMI were significantly and positively associated with knowledge level about risk factors of CVD [4]. In addition, majority of adolescents do not have sufficient knowledge about major cardiovascular diseases risk factors [5]. Stroebele et al. (2011) were of the view that Knowledge of CVD risk factors is necessary for making informed decisions regarding participation in or maintaining behaviors that may reduce the risk of CVD [6]. Bandura (2004) also noted that Knowledge of health risks and benefits creates the precondition for change [6]. All these studies showed that assessment of common risk factors and patients' knowledge about CVD is needed for effective CVD treatment and prevention strategies.

\section{Methodology}

The study site was Kitwe Teaching Hospital (KTH) which is found along Kuomboka drive, in Parklands, Kitwe, Zambia. The target population was persons aged 18 years or older who presented to Kitwe Teaching Hospital's internal medicine or surgical department for medical services. This included both inpatients and outpatients who may or may not have had cardiovascular disease. This study was carried out after approval had been given by the Ethics Review Committee at Tropical Disease Research Centre of Ndola, the Public Health Team at CBU and The Management at Kitwe Teaching Hospital. Furthermore, verbal consent was obtained from respondents before collecting data. Also, confidentiality was maintained by omitting the names of the respondents. Lastly, the questionnaire was interpreted in the patient's own language for those who were not able to read, write and speak English language. A standardized questionnaire that had both closed and open ended questions was used to collect data from cases (patients with cardiovascular disease) and controls (patients with no cardiovascular disease). The questionnaire had three parts. Part A consisted of questions on demographics, Part B had questions that were assessing cardiovascular risk factors and Part $\mathrm{C}$ had questions that assessed the level of knowledge of cardiovascular disease. Cleaned data was entered into the Statistical Package for Social Sciences (SPSS) version 16.0 were all the statistical analysis were performed. The Pearson Chi-square test was used to determine associations in bivariate analyses at the $5 \%$ significance level. Likewise, independent factors associated with cardiovascular disease were established using the Binary logistic regression analysis. The unadjusted odds ratios (OR) and Adjusted odds ratios (AOR) were calculated and the confidence interval (CI) was set at $95 \%$.

\section{Results}

A total of 148 people participated in the study comprising 74 cases (those with cardiovascular disease) and 74 controls (those with no cardiovascular disease). The mean age of the respondents (both cases and controls) was 45.9 years with a range of 20 to 83 years and standard deviation of 14.66. 40 and 50 years were the modes.

Table 1. Frequency distribution of age group, gender, level of education and occupation for cases and controls.

\begin{tabular}{|c|c|c|c|c|c|}
\hline \multirow[t]{2}{*}{ Age group } & \multicolumn{2}{|c|}{$\begin{array}{l}\text { Participant } \\
\text { classification }\end{array}$} & \multirow[t]{2}{*}{ Total } & \multirow[t]{2}{*}{ Chi-square } & \multirow[t]{2}{*}{$P$ value } \\
\hline & Cases & Controls & & & \\
\hline $18-25$ & 1 & 7 & 8 & & \\
\hline $26-45$ & 33 & 41 & 74 & & \\
\hline $46-60$ & 25 & 16 & 41 & 8.340 & 0.039 \\
\hline Above 60 & 15 & 10 & 25 & & \\
\hline Total & 74 & 74 & 148 & & \\
\hline \multicolumn{6}{|l|}{ Gender } \\
\hline Male & 29 & 39 & 64 & 0.991 & 0.319 \\
\hline Female & 45 & 35 & 84 & & \\
\hline Total & 74 & 74 & 148 & & \\
\hline \multicolumn{6}{|l|}{$\begin{array}{l}\text { Level of } \\
\text { education }\end{array}$} \\
\hline $\begin{array}{l}\text { No formal } \\
\text { education }\end{array}$ & 9 & 1 & 10 & & \\
\hline Primary school & 28 & 36 & 64 & 7.400 & 0.060 \\
\hline Secondary school & 18 & 18 & 36 & & \\
\hline $\begin{array}{l}\text { Tertiary } \\
\text { Education }\end{array}$ & 19 & 19 & 38 & & \\
\hline Total & 74 & 74 & 148 & & \\
\hline \multicolumn{6}{|l|}{ Occupation } \\
\hline Employed & 15 & 18 & 33 & & \\
\hline Self employed & 12 & 23 & 35 & & \\
\hline Unemployed & 34 & 18 & 52 & 8.796 & 0.032 \\
\hline Other & 13 & 15 & 28 & & \\
\hline Total & 74 & 74 & 148 & & \\
\hline
\end{tabular}

The table above (Table 1) shows that most participants were aged between 26 and 60 for both cases (78\%, within cases) and controls (77\%, within controls) with a chisquare and $\mathrm{p}$ value of 8.340 and 0.039 respectively showing that there was a significant relationship. The table also reviews that there were more females than the males for both cases and controls. Females were $61 \%$ within cases and 53\% within controls and the overall amounted to $57 \%$. Chi-square and $\mathrm{p}$ value for gender distribution were 0.991 and 0.319 in that order. The level of education as tabulated above evinces that an overall of $43.2 \%$ participants had education up to primary school level which they either completed or dropped out, $34.3 \%$ had attended secondary (both completed and drop outs), $26 \%$ had tertiary education and $7 \%$ had no formal education. Pearson Chi - square: 7.400 and $\mathrm{P}$ - value: 0.060 . Table 1 further shows concludes that $35.1 \%$ of the participants were unemployed while $18.9 \%$ (Others) were either retired or students currently in school. Pearson Chi - square: 8.796 and $\mathrm{P}$ - value: 0.032 . 
Table 2. Risk factors exposure rate calculated using odds ratio (OR) $n=148$.

\begin{tabular}{|c|c|c|c|c|c|c|}
\hline No & Risk factor & Casesf & Controlf & OR & CI-95\% L/U limit & P value \\
\hline \multirow[t]{5}{*}{1} & Age (year) & & & & & \\
\hline & $18-25$ & 1 & 7 & & & 0.039 \\
\hline & $26-45$ & 33 & 41 & & & \\
\hline & $46-60$ & 25 & 16 & & & \\
\hline & Above 60 & 15 & 10 & & & \\
\hline \multirow[t]{3}{*}{2} & Family History of CVD & & & & & \\
\hline & Yes & 56 & 41 & 2.504 & $1.242 / 5.050$ & 0.009 \\
\hline & No & 18 & 33 & & & \\
\hline \multirow[t]{3}{*}{3} & Smoking & & & 1.587 & $0.731 / 3.448$ & 0.241 \\
\hline & Yes & 20 & 14 & & & \\
\hline & No & 54 & 60 & & & \\
\hline \multirow[t]{3}{*}{4} & Alcohol Consumption & & & 1.387 & $0.725 / 2.654$ & 0.322 \\
\hline & Yes & 37 & 31 & & & \\
\hline & No & 37 & 43 & & & \\
\hline \multirow[t]{3}{*}{5} & Hypertension & & & 59.143 & $20.122 / 173.833$ & 0.00 \\
\hline & Yes & 60 & 5 & & & \\
\hline & No & 14 & 69 & & & \\
\hline \multirow[t]{3}{*}{6} & High blood cholesterol & & & 4.364 & $0.894 / 21.293$ & 0.049 \\
\hline & Yes & 8 & 2 & & & \\
\hline & No & 66 & 72 & & & \\
\hline \multirow[t]{3}{*}{7} & Diabetes Mellitus & & & 3.725 & $1.722 / 8.057$ & 0.001 \\
\hline & Yes & 31 & 12 & & & \\
\hline & No & 43 & 62 & & & \\
\hline \multirow[t]{3}{*}{8} & Regular exercise & & & 0.575 & $0.287 / 1.150$ & 0.116 \\
\hline & Yes & 20 & 29 & & & \\
\hline & No & 54 & 45 & & & \\
\hline \multirow[t]{3}{*}{9} & Balanced diet & & & 0.890 & $0.456 / 2.195$ & 1.738 \\
\hline & Yes & 46 & 48 & & & \\
\hline & No & 28 & 26 & & & \\
\hline \multirow[t]{3}{*}{10} & Regular weight check & & & 0.897 & $0.471 / 1.710$ & 0.742 \\
\hline & Yes & 35 & 37 & & & -.027 \\
\hline & No & 39 & 37 & & & \\
\hline
\end{tabular}

Table 3. Knowledge score.

\begin{tabular}{llllll}
\hline & \multicolumn{4}{c}{ Knowledge Score Classification } & \multirow{2}{*}{ Total } \\
\cline { 2 - 5 } & & Low & Average & High & \\
\hline Participant & Cases & 4 & 34 & 36 & 74 \\
Classification & Controls & 8 & 50 & 16 & 74 \\
Total & & 12 & 84 & 52 & 148 \\
\hline
\end{tabular}

Table 3 shows the knowledge score of participants of both cases and controls. Knowledge was assessed by use of two questions, the first question was about the risk factors of cardiovascular diseases patients know. This question had 9 subquestions. Question 2 assessed the participants on the signs and symptoms of cardiovascular diseases they know and had 10 subquestions. This summed up to 19 questions of assessing the knowledge of participants. A score of 0-9 was classified as low, 10-14 as average score and those that scored 15-19 were classified to have a high score. Only $35 \%$ overall had a high knowledge score. The remaining $65 \%$ either had a low or average score. Within cases only $49 \%$ had high knowledge score, $46 \%$ average and $5 \%$ low score. Within controls only $22 \%$ had high knowledge score, 68\% average and 10\% low score. Pearson Chi Square 12.073 and $\mathrm{P}$ value 0.002 .
Table 4. Cross tabulation between knowledge score and age group.

\begin{tabular}{llllll}
\hline & & \multicolumn{3}{l}{ Knowledge Score Classification } & \multirow{2}{*}{ Total } \\
\cline { 3 - 5 } & & Low & Average & High & \\
\hline \multirow{4}{*}{ Age Group } & $18-25$ & 1 & 6 & 1 & 8 \\
& $26-45$ & 4 & 43 & 27 & 74 \\
& $46-60$ & 2 & 19 & 20 & 41 \\
Total & Above 60 & 5 & 16 & 4 & 25 \\
\hline
\end{tabular}

The above table (table 4 ) shows that $19 \%$ of those within the age group 18 to 45 had high knowledge score, while $33 \%$ had an average score and $3 \%$ had a low knowledge score. $16 \%$ of those within the age group 46 to 83 had high knowledge score, while $24 \%$ had an average score and $5 \%$ had a low knowledge score. The Pearson Chi value 13.236, P value 0.039 .

\section{Discussion}

This study had a total of 148 participants comprising 74 cases (those with cardiovascular disease) and 74 controls (those with no cardiovascular disease). The mean age of the 
respondents (both cases and controls) was 45.9 years with age range of 20 to 83 years and standard deviation of 14.66. The mode ages were 40 and 50 years.

The majority of the participants were aged between 26 and 60 years for both cases $(78 \%$, within cases) and controls (77\%, within controls). This result shows that CVDs are more prevalent in this age group and among females. However, several studies [7] have revealed that the disease is more prevalent in the older adults (those above 50) which is partially true when compared to the findings of this study. Several reasons could contribute to the increased prevalence of CVD in this age group and among females in Kitwe. For one thing, individuals aged between aged between 26 and 45 years usually engage in risk behaviours such as smoking, drinking, lack of physical exercise and poor eating habits. The last two are especially true for women who are mostly not employed and mostly housekeepers. For another, genetic predisposition makes these individuals susceptible to CVDs. This study found that $65.5 \%$ of the participants had a family history of CVDs. The lower prevalence of CVD in those above 60 years could be due to the fact that individuals who get sick between the ages of 26 and 60 do not survive long enough because of the fatality of the disease.

With regard to marital status, most of the participants were married $(61.5 \%$ overall) while the minority $(11.5 \%)$ belonged to the group Other (Separation, widow and widower). The Pearson Chi - square and $\mathrm{P}$ values were 1.686 and 0.640 respectively. Furthermore, $43.2 \%$ of the participants had education up to primary school level which they either completed or dropped out, $34.3 \%$ had attended secondary (both completed and drop outs), 26\% had tertiary education and $7 \%$ had no formal education. Pearson Chi - square: 7.400 and $P$ - value: 0.060 . The low levels of education could explain while most of the participants are unemployed (35.1\%). However, this could also be due to most participants being female and hence housekeepers. High unemployment levels among females was attributed to them being housekeepers [8]. In this study, there was a significant relationship between level of education and occupation. Pearson Chi square: 64.191 and $P$ value: 0.000.

This study showed that the following factors were independently associated with cardiovascular disease: age, family history of CVDs, smoking, alcohol consumption, hypertension, high blood cholesterol, diabetes mellitus, lack of physical exercise, lack of balanced diet and inability to control weight. These factors have also been identified in several studies that have been conducted across the world [4, 9-12].

Age group was significantly associated with developing CVD, P value: 0.039 . The age group $26-60$ within cases was strongly associated with CVD (78\%). When divided into two smaller groups, CVD was more common in the age group $26-45$ (46\%, within cases) than in the group $46-60$ (34\%, within cases). This finding is closely similar to what was found, were the largest number of patients with CVD (cases) were present in the age group of 51-60 years (40\%), followed by 41-50 years (27.4\%) [13].

Hypertension and diabetes are the leading risk factors for atherosclerosis, heart attacks, and strokes [14]. Another study was conducted and it was identified that diabetes mellitus and hypertension as the major risk factors for cardiovascular disease [15]. This study has also observed that hypertension and diabetes mellitus were significantly associated with the risk of developing CVD. High blood pressure was prevalent among $44 \%$ of the patients included in this study ( $81 \%$ of the cases and only $7 \%$ of the controls). Similar results have been produced by Smith, were a total of $43.7 \%$ of patients included in their study $(72.3 \%$ of cases and $32.8 \%$ of controls) were hypertensive [6]. Reference [11] also found that hypertension was present in $62 \%$ of cases and $20 \%$ in the controls. However, the odds ratios of a hypertensive patient developing CVD that were reported in these studies were lower than those found in this study. The former study reported an odd of 7 while the latter reported an odd of 6.5 . In the current study, the odds of a person who had hypertension having CVD was 59.143 times more than a person without hypertension (OR 59.143, 95\% CI 20.122173.833 and adjusted odds ratio was AOR 97.289, 95\% CI $24.479-386.662)$. Likewise, diabetes was prevalent among $29 \%$ of the patients $(42 \%$ within cases and $16 \%$ within controls). This is slightly lower than what kapoor et al [11] found (50\%). The odds of a diabetic patient developing CVD was 3.725 times (OR 3.725, 95\% CI 1.722 - 8.057; AOR $6.033,95 \%$ CI 1.630 -22.332) more than a non - diabetic patient. This is similar to the findings of kapoor [11] in which diabetic patient had an odd of 4.5 (OR; 4.5, 95\% CI; 2.4-8.7) of developing CVD. These finding are scary and clearly highlights the need to conduct a prospective study to assess patients' adherence to antihypertensive and antidiabetic medication and whether this is related to decreasing risk of developing CVDs.

Family history of CVD, smoking and alcohol consumption were also significantly associated with risk of developing cardiovascular disease. $66 \%(76 \%$ within cases and 55\% within controls) of the patients had a positive family history of CVD, 23\% (27\% within cases and 19\% within controls) admitted to smoking and $46 \%$ (50\% within cases and $42 \%$ within controls) consumed alcohol. These findings are similar to the findings of Rohit and Atul [13]. In this study a person with a family history of CVD was 2.504 times more likely to develop the disease than a person within family history (OR 2.504, 95\% CI 1.242-5.050; AOR 0.862, 95\% CI 0.244-3.041). Individuals who smoked and consumed alcohol were 1.587 times (OR 1.587, 95\% CI 0.731-3.448; AOR $0.746,95 \%$ CI $0.164-3.403$ ) and 1.387 times (OR 1.387, 95\% CI 0.725 -2.654; AOR 3.392, 95\% CI 0.833 - 13.823) more likely to develop CVD when compared to those who neither smoked nor drank alcohol. These finding are lower than those found by kapoor [11], in which the odds ratios were: alcohol consumption (OR; 14.6, 95\% CI; 6.4-33.3), smoking (OR; 13.6, 95\% CI; 6.6-27.8), and family history (OR; 5.3, 95\% CI; 2.8-9.9). In this study it was found that $27 \%$ and $50 \%$ of CVD patients smoke and consume alcohol respectively, is a serious source of concern. Thus health workers still have a lot of work to do in educating patients 
about CVD risk factors, and consequences of smoking and drinking on their health.

In addition, the study revealed that having high blood cholesterol was also associated with high odds of developing CVD, 4.364 times (OR 4.364, 95\% CI 0.894 - 21.293; AOR $4.482,95 \%$ CI $0.242-82.888$ ) when compared with a person who has normal blood cholesterol. Overall 7\% (11\% within cases and 3\% within controls) of the patients had high blood cholesterol. Reference [16] identifies high cholesterol levels as a risk factor for CVD. In their study, they observed that participants who were overweight were more likely to have raised cholesterol levels. A 2007 study reported from the Women's Health Study that high levels of total cholesterol is significantly associated with an increased risk for ischemic stroke, even in women who are otherwise healthy [16]. Thus, cholesterol levels should be controlled in individuals who have abnormal levels so as to reduce their chances of developing CVD or its complications.

Over and above, this study found that physical exercise, having a balanced diet and regular weight checks (and weight control) were significantly associated with smaller odds of developing CVDs. A person who was active physically (physical exercise) and had a balanced diet had odds of 0.575 times (OR 0.575, 95\% CI 0.287-1.150; AOR 1.849, 95\% CI $0.507-6.751)$ and 0.890 times (OR $0.890,95 \%$ CI $0.456-$ 2.195; AOR 1.451, 95\% CI $0.404-5.214)$. An individual who regularly checked his/her weight and controlled it also had slim odds of 0.897 (OR 0.897, 95\% CI $0.471-1.710$; AOR 0.593, 95\% CI $0.177-1.990$ ) of developing the disease. Several studies have highlighted the benefits on physical exercise, balanced diet and weight control [14-8]. Physical activity is necessary for the metabolic and cardiovascular benefits [8]. Physical activity can slow the initiation and development of diabetes and the sequence of CVD through its effect on body weight, insulin sensitivity, glycaemic control, blood pressure, fibrinolysis, endothelial function and inflammatory defence systems. Diet plays a vital role in human health [14]. However, (Allison and Mattes 2009), warns that over-consumption of food may lead to excessive energy intake and result in overweight and obesity, which is closely associated with the onset of diabetes mellitus [14].

Apart from the considered aspects, this study also assessed the patients' level of knowledge of CVDs risks factors, signs and symptoms. The study revealed that Only $35 \%$ overall had a high knowledge score. The remaining $65 \%$ either had a low or average score. Within cases only $49 \%$ had high knowledge score, $46 \%$ average and $5 \%$ low score. Within controls only $22 \%$ had high knowledge score, $68 \%$ average and $10 \%$ low score (Pearson Chi Square 12.073 and $\mathrm{P}$ value 0.002). Similar findings have been reported in other parts of the world $[9,17,4]$. This finding is alarming because at this stage, patients should be well informed of the disease, in order for them to know how to protect themselves.

The study also revealed that there was a significant relationship between Age and Level of knowledge (Pearson Chi 13.236, P value 0.039). 19\% of those within the age group 18 to 45 had high knowledge score, while $33 \%$ had an average score and $3 \%$ had a low knowledge score. $16 \%$ of those within the age group 46 to 83 had high knowledge score, while $24 \%$ had an average score and $5 \%$ had a low knowledge score. This result is almost similar to Al-Nafinsi's [4] findings. Gender was also significantly related to the level of knowledge ( $\mathrm{P}$ - value 0.007$)$. However, level of education did not show a significant relationship with knowledge score (P value: 0.276).

\section{Study Limitation}

One of the major limitation in the study was financial as the study was not funded. Another one was time. The study was conducted during school, therefore not enough time was left to collect data.

\section{Conclusion}

The study has identified the major risk factors for CVD at Kitwe Teaching Hospital and these are increased age, hypertension, diabetes mellitus, family history of CVD, high blood cholesterol, smoking, alcohol consumption, physical inactivity, lack of balanced diet and lack of body weight control. Of these, hypertension and diabetes were associated with increased odds of developing CVDs. Furthermore, physical activity, having a balanced diet and body weight control were associated with reduced chances of developing the disease. This study also revealed that there was poor knowledge of CVDs risk factors as well as its signs and symptoms among patients.

The results of this study provide important information for policy makers, to introduce educational programs both at health facilities and within the community that aim to educate patients and members of the community about CVDs and other non - communicable diseases.

\section{References}

[1] Ministry of Health (2012). Zambian Strategic Plan 2013-2016: NON-COMMUNICABLE DISEASES AND THEIR RISK FACTORS.

[2] Mulenga M. M (2016). Government's Health Policy Response to Non- Communicable Diseases in Zambia. The University of Zambia: School of Medicine. A dissertation submitted in partial fulfilment of the requirements for the degree of Master of Public Health - Health Policy and Management.

[3] Siziya S, Rudatsikira E, Babaniyi O, Songolo P, Mulenga D, et al. (2012) Prevalence and Correlates of Hypertension among Adults Aged 25 Years or Older in a Mining Town of Kitwe, Zambia. J Hypertens 1:105. doi: 10.4172/21671095.1000105 .

[4] Al-Nafisi. H (2017). Knowledge of Cardiovascular Disease and its Risk Factors among the Community in Kuwait. MedCrave Group LLC 
[5] Adhikari N, Sapakota KP, Adhikari S (2018). Cardiovascular Diseases (CVDs) Risk Attitude and Knowledge Level of Major Risk Factors for Cardiovascular Diseases among 15-19 Years Eleventh and Twelfth-Grade Students of Lekhnath Municipality. J Community Med Health Educ 8: 584. doi: 10.4172/2161-0711.1000584

[6] Linda M. Smith (2015). Educational Intervention: Effects on Heart Disease Risk Factor Knowledge Among African Americans. Walden Dissertations and Doctoral Studies Collection at ScholarWorks, Walden University.

[7] Joel Negin, Robert Cumming, Sarah Stewart, et al (2011), Risk Factors for Non-communicable diseases in older adults in rural Africa, Tropical Medicine International Health, vol 16, doi: 10.1111/j.1365-3156.2011.02739. x

[8] Huda Nimer Mohammad Lahham (2009). Cardiovascular Diseases and Risk Factors Among Diabetic Patients in Nablus District, West Bank, Palestine: case-control study. Thesis submitted in Partial Fulfillment of the Requirements for the Degree of Master in Public Health, Faculty of Graduate Studies, An-Najah National University, Nablus, Palestine.

[9] Boateng D, Wekesah F, Browne JL, Agyemang C, AgyeiBaffour P, Aikins Ad-G, et al. (2017) Knowledge and awareness of and perception towards cardiovascular disease risk in sub-Saharan Africa: A systematic review. PLoS ONE 12 (12): e0189264. (Document was retrieved from https://doi.org/10.1371/journal.pone.0189264 on 28th April, 2018).

[10] Ministry of Health (2016). ZAMBIA NATIONAL HEALTH STRATEGIC PLAN 2017 - 2021

[11] Kapoor. R, Vyas. S, Patel. P, Mehta. H, Mehta. P, Modi. J, and Nair. S (2013). A case-control study of risk factors for ischemic heart disease in patients attending tertiary care hospitals in India. South East Asia Journal of Public Health 2013; 3 (1): 57-60.

[12] El-Hajj. M, Salameh. P, Rachidi. S, Al-Hajje. A, Lahoud. N and Hosseini. H (2018). Stroke risk factors: a hospital-based case-control study in Lebanon. Journal of the Royal Society of Medicine Open; 8 (6) 1-10, DOI: $10.1177 / 2054270417713692$.

[13] Ram RV, Trivedi AV (2012). Behavioural risk factors of coronary artery disease: A paired matched case control study. J Cardiovasc Dis Res 2012; 3: 212-7.

[14] Thanh Chung Nguyen (2016). A Case-Control Study of Risk Factors for Type 2 Diabetes Mellitus in Vietnam. Thesis presented for the Degree of Doctor of Philosophy at Curtin University.

[15] Schweigman K., Eichner J., Welty K. T., and Zhang Y (2006). Cardiovascular Disease Risk Factor Awareness in American Indian Communities: The Strong Heart Study. Center for American Indian Health Research, University of Oklahoma Health Sciences Center, Oklahoma City, Oklahoma.

[16] Muula S. A, Rudatsikira. M, Babaniyi. O, Songolo. P, Mulenga. D and Siziya S. (2012). Factors Associated with High Cholesterol levels in Lusaka, Zambia: A CommunityBased Study. Medical Journal of Zambia, Vol. 39, No. 4 (2012).

[17] Bhawana D. and Neetu S. (2015). Public knowledge of cardiovascular diseases and its risk factors among early adulthood: A review. International Journal of Applied $\begin{array}{llll}\text { Research } & 2015 ; & 1 & \text { (12): }\end{array}$ https://www.researchgate.net/publication/299469397 (website accessed on 28th April, 2018). 